\title{
Atomistic simulations of dopamine diffusion dynamics on a pristine graphene surface
}

\author{
Qizhang Jia, Cheng Yang, B. Jill Venton, and Kateri H. DuBay* \\ Department of Chemistry, University of Virginia, Charlottesville, Virginia 22904 \\ E-mail: dubay@virginia.edu
}

\section{Abstract}

Carbon microelectrodes enable in vivo detection of neurotransmitters, and new electrodes are being developed to optimize the carbon surface. However, the work is mainly empirical and there have not been corresponding theoretical studies using molecular-level simulations of the diffusion and orientation of neurotransmitters near these surfaces. Here, we employ molecular dynamics simulations to investigate in atomistic detail the surface diffusion of dopamine (DA), its oxidation product dopamine-o-quinone (DOQ), and their protonated forms on the pristine basal plane of flat graphene. All DA species rapidly adsorb to the surface and remain adsorbed for the full length of the equilibrium simulations, even without a holding potential or graphene surface defects. The diffusivities of the adsorbed and the fully solvated DA are similar, and all molecular diffusion on the surface is slower than that of an adatom of comparable molecular weight. The protonated species diffuse more slowly than their corresponding neutral forms, and the oxidized species diffuse more rapidly. The underlying hexagonal graphene structure has little influence over the molecular adsorbate's lateral position. The vertical placement of the amine group on dopamine is highly dependent upon its charge, and the protonated amine prefers to be above the surface near the solvating waters. Solvation has a large effect on surface diffusivities when diffusion is compared to that in a vacuum. These are the first results of molecular dynamics simulations of dopamine diffusion at the aqueous-graphene interface, and they show that dopamine diffuses quickly on graphene surfaces, even without an applied potential. These calculations provide a basis for future simulations to predict the behavior of neurotransmitter diffusion on advanced carbon materials electrodes.

KEYWORDS: dopamine, graphene, fast scan cyclic voltammetry, molecular dynamics, diffusion dynamics, microelectrode, nanomaterials

\section{Introduction}

In vivo electrochemistry is a powerful quantitative analytical technique that can measure the oxidation or reduction of target compounds within living organisms. ${ }^{[1] 3}$ In these experiments, a biocompatible carbon electrode is inserted into live tissue to observe transient changes of key biochemical species, such as neurotransmitters, ${ }^{4}$ signaling molecules,,$\frac{5}{5}$ and physiological metabolites ${ }^{[16] 9]}$ - species whose biological activities depend on the timing and location of their release. One such species, dopamine (3,4-dihydroxyphenethylamine, DA), is a critical neurotransmitter in the central nervous system and controls reward-motivated behaviors. ${ }^{10[12]} \mathrm{DA}$ is a common target for in vivo electroanalytical detection and rapid electrochemical studies of dopamine have improved our understanding of its role underlying positive reinforcement, decision making, and addiction. $\frac{13[18}{118}$ Carbon-based microelectrodes are 
widely used for the in vivo electrochemical detection of $\mathrm{DA}^{13|14| 18 \mid 19}$ and similar species due to their fast electron transfer rates, biocompatibility, and low costs. $\frac{13 \mid 20}{22}$ Coupled with fastscan cyclic voltammetry (FSCV), which uses rapid potential sweeps to repeatedly oxidize and reduce analytes of interest, carbon microelectrodes have been able to measure neurotransmitters in vivo with millisecond temporal resolution. $14|18| 23$

The first generation carbon microelectrodes consisted primarily of carbon fibers of disordered graphite, $13|15| 20|24| 25]$ often treated to enhance electrochemical activity. Beveling or physically polishing a graphite disk increases the ratio of edge to basal planes, while electrochemical pre-treatments increase the frequency of electrochemically active defects and graphite oxide moieties on the surface. ${ }^{26}$ Such carbon surface features enhance the adsorption of catecholamines and facilitate the subsequent oxidation and reduction during electrodetection. $13[27][32$

At the same time, recent local electrochemical imaging studies by Unwin and coworkers have emphasized that even the basal planes of pristine highly-ordered pyrolytic graphite (HOPG) and carbon nanotubes (CNTs) display significant electrochemical activity towards catecholamines. ${ }^{33}$ Indeed, even early work on freshly fractured glassy carbon microelectrodes showed that pristine basal plane graphene sheets exhibited significant DA adsorption and activity, although electrochemical activity could still be enhanced after certain pre-treatments. 30

In more recent years, a wide variety of advanced carbon electrodes have been developed, including graphene oxide nanoribbons, ${ }^{34} \mathrm{CNT}$ yarn microelectrodes (CNTYMEs), $\stackrel{35}{\text { carbon }}$ nanospikes,, 36 cavity carbon nanopipettes,, $37 / 38$ laser activated CNTYMEs,, 15 and microelectrodes coated with carbon nanohorns or nanodiamonds. ${ }^{39}$ These novel microelectrodes have improved sensitivity, temporal resolution, and analyte specificity.

Although investigations of these novel carbon microelectrodes have led to reliable general principles governing the relationship between electrode structure and its function, 36 details are still lacking on the underlying molecularscale determinants of analyte adsorption and desorption, oxidation and reduction catalysis, and analyte dynamics in solution and on the electrode surface. Indeed, even the simplest questions regarding the diffusivity of catecholamines on a pristine carbon microelectrode surface have not yet been comprehensively studied.

While catecholamine adsorption and desorption occurs on the $\mu$ s to $\mathrm{s}$ timescale, 43144 diffusion of solvated catecholamines to the surface is more rapid ${ }^{29}$ with flow injection experiments suggesting a solvated diffusion constant of $D=0.6 \times 10^{-5} \mathrm{~cm}^{2} / \mathrm{s}$. 45 Diffusion constants for catecholamines adsorbed on carbon microlectrode surfaces have not been characterized, although evidence suggests that these could also be much faster than the adsorption/desorption times. An MD study of DA on a $\mathrm{TiO}_{2}$ surface reported that, at a surface coverage close to the saturation limit, the DA distribution over surface facets of $70 \mathrm{~nm}^{2}$ had equilibrated by $2 \mathrm{~ns} . \stackrel{46}{ }$ Since very local surface features can influence nearby electrochemical activity, 13136 and DA dwell time on the surface has been estimated to be more than 0.2 seconds, $\frac{43}{4}$ characterizing the surface dynamics of adsorbed analytes is essential for understanding microelectrode function. Indeed, recent work postulated the existence of electroactive surface domains separated by inactive domains and showed that the dependence of oxidation wave peak current intensities on FSCV scan rate could be explained by the time it takes for adsorbed DA to move between them during the scan window. $\frac{47}{4}$ Although previous computational studies investigated the strength of the energetic interactions between graphene and DA in different orientations ${ }^{48}$ as well as the behavior of atomic adsorbates ${ }^{49 / 50}$ and $\mathrm{C}_{60}$ molecules ${ }^{5152}$ on graphene surfaces, the diffusion of DA and DOQ on graphene has not been previously simulated.

In this paper, we use molecular dynamics (MD) to investigate the surface dynamics of various DA species on the basal plane of pristine graphene. In Section 2, we describe our 
modeling approach for simulating DA, its oxidized form dopamine-o-quinone (DOQ), and their protonated counterparts on a pristine flat graphene surface. In Section 3, we quantify the differences in diffusion between the DA species and the lateral and vertical distributions of their constituent moieties on the surface, comparing our results to those of a structureless adatom of the same mass. Finally, we discuss our findings and their implications in Section 4 and conclude in Section 5. These studies show how dopamine diffuses on a pristine graphene surface, which is crucial for understanding dopamine-electrode interactions and designing better materials for electrodes.

\section{Methods}

We employed atomistic MD simulations on 3D periodic systems that contained a single adsorbate, a flat graphene surface, and TIP3P water molecules.

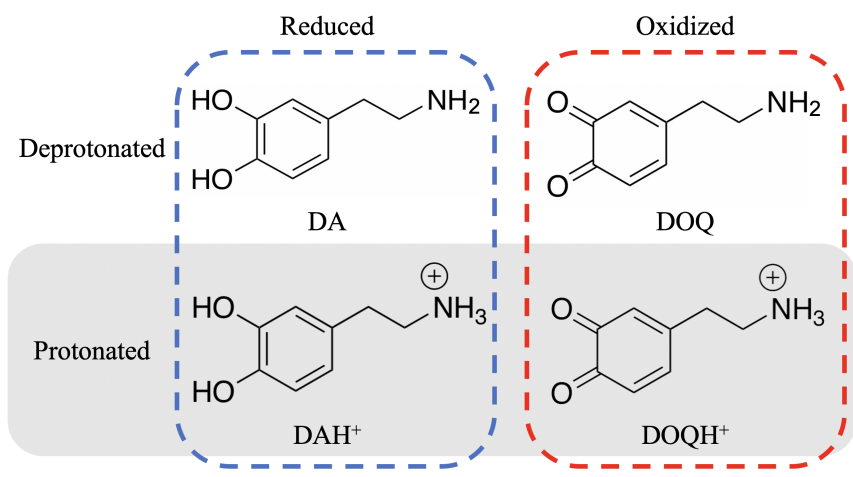

(a)

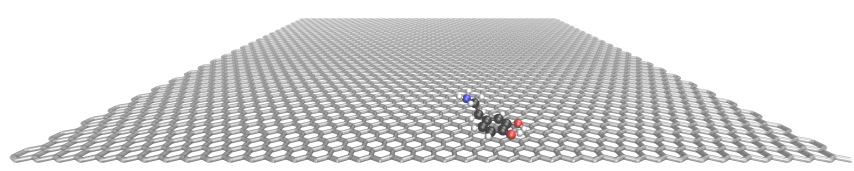

(b)

Figure 1: DA species and graphene. (a) Various dopamine (DA) species: DOQ is the oxidized product of $\mathrm{DA}$, and $\mathrm{DAH}^{+}$and $\mathrm{DOQH}^{+}$are the protonated species of DA and DOQ, respectively. (b) An atomistic representation of DA is shown adsorbed onto the flat graphene surface.

Dopamine and Adatom Adsorbates. The oxidative product of DA is dopamine- $O$ quinone (4-(2-Aminoethyl)-3,5-cyclohexadiene1,2-dione, DOQ), which is an important in- termediate product in DA metabolism. $\frac{53}{\mathrm{pH}}$ is well regulated in the human body, with an arterial pH between 7.36 and 7.44 and an intracellular $\mathrm{pH}$ of approximately $7.2 . \sqrt[54]{5}$ The three $\mathrm{p} K_{\mathrm{a}}$ 's of DA are estimated to be $\mathrm{p} K_{\mathrm{a}, 1}=8.71$, $\mathrm{p} K_{\mathrm{a}, 2}=10.90$, and $\mathrm{p} K_{\mathrm{a}, 3}=13.68 .{ }^{[55]}$ As a result, at physiological $\mathrm{pH}, \mathrm{DA}$ and DOQ primarily exist in their singly protonated states, as $\mathrm{DAH}^{+}$ and $\mathrm{DOQH}^{+} . \underline{56}$

In our modeling, we represented DA, DOQ, and their singly protonated species atomistically (see Fig. 17a) and assigned optimized charge distributions for each species (see SI for additional details). Counter-charges for the protonated species were added in the form of $\mathrm{Cl}^{-}$ions. DA is a catecholamine containing three key moieties: the side-chain amine, the aromatic ring, and two ortho-hydroxyl groups. $\stackrel{53}{ }$ We refer to these moieties as "amine," "ring," and "diol" or "quinone," the last depending on whether it is DA or DOQ. In addition to the DA species, we also model a chargeneutral atomic adsorbate with the same molar mass as dopamine (153.18 a.u.) for comparison purposes, which we refer to as "adatom(DA)."

Flat Graphene Surface. A single-layered pristine flat graphene surface was used in our simulations to represent a conventional carbon fiber microelectrode surface. Fig,1(b) illustrates the simulated graphene system. The decision to model just a single layer of graphene was made after comparisons between the surface diffusion dynamics on single- and triplelayered graphene in the solvated systems displayed no significant differences (see SI for more details). The graphene carbon atoms were kept fixed in order to mimic a stationary bulk phase carbon electrode while minimizing complications that could be introduced by other approaches to keep graphene stationary. This choice is discussed further in the SI. The simulation box extended both $15 \AA$ above and $15 \AA$ below the carbon surface in the $z$-direction. Finally, after simulating a series of different dimensions for the flat graphene surface to test the influence of the system size on the resulting DA diffusion constants, we chose the largest simulation size, with graphene surface dimensions of $98.2 \times 97.8 \AA^{2}$, to minimize the finite 
system-size effects. A full discussion of the finite system size effect can be found in the SI, and estimates of the infinite system size diffusion constant, $D_{\infty}$, are made there for DA and adatom(DA) by fitting the results from five different system sizes and extrapolating to the infinite case. This extrapolation can be seen in Figure $\mathrm{S} 1$, and the resulting $D_{\infty}$ values are reported in Table S2.

Simulation Details. Atomistic MD simulations were performed in the canonical ensemble at $300 \mathrm{~K}$ for $5 \mathrm{~ns}$ using a Nosé-Hoover thermostat. The equations of motion were integrated using a standard velocity-Verlet algorithm with a time step of $1 \mathrm{fs}$. Ten independent MD simulations were performed for all results presented below, using the Largescale Atomic/Molecular Massively Parallel Simulator (LAMMPS) .57 Additional simulation details can be found in the SI.

The interactions between the adsorbates and the flat graphene surface were modeled using the classical OPLS-AA force field,, 58 which has been validated for similar systems in prior studies. In Lazar et al., the enthalpies of a series of small organic molecules adsorbing onto the graphene surface were calculated using OPLS$\mathrm{AA}$, and the results correlated well to the experimentally determined adsorption enthalpies, although they underestimated the experimental values by about $1.9 \mathrm{kcal} / \mathrm{mol}{ }^{59}$ In Björk et al., the accuracy of various computational approaches in calculating binding energies per carbon atom was assessed for a series of $\pi$ conjugated systems adsorbed on the graphene surface, and OPLS-AA was again able to reproduce the experimentally observed trend. 60

For simplicity and clarity, no external voltage was applied in the MD simulation results presented in this paper. Although the applied potential exerts significant influence over adsorbed DA, the potential typically scans rapidly from $-0.4 \mathrm{~V}$ to $1.3 \mathrm{~V}$ (vs. an $\mathrm{Ag} / \mathrm{AgCl}$ reference) and back, with the limits of that scan chosen strategically to maximize DA adsorption at the holding potential of $-0.4 \mathrm{~V}$, cover the full range of the oxidation and reduction peaks, and optimize electrode performance via a surface activation at the potentials $>1.0 \mathrm{~V} .{ }^{23}$ Moreover, the location of the peaks actually shift with FSCV scan rate. 23 Thus, a comprehensive assessment of the influence of the applied voltage on DA dynamics is complex and lies outside the scope of this first investigation of DA dynamics on the graphene surface.

A small number of simulations were also run without solvent, in order to isolate the influence of the surface on adsorbate diffusion. In these cases, only neutral DA and DOQ were modeled and simulations were run within the NVE ensemble.

\section{$3 \quad$ Results and Discussions}

\subsection{DA species adsorb rapidly and remain on the pristine graphene surface.}

All simulations were initially set up with the analyte fully dissolved some distance above the graphene surface. Adsorption and desorption timescales were estimated for the DA and DOQ concentrations within our simulations, using the kinetic model proposed by Bath et al. 35161 $^{361}$ Desorption events, for both DA and DOQ, were estimated to take place on the timescale of seconds, while adsorption events were estimated to take place several orders of magnitude more rapidly (see SI for details). Desorption events are thus not expected to occur on the timescales accessible in our equilibrium MD simulations. Indeed, in all simulations, the DA species adsorbed to the surface within the first $0.2 \mathrm{~ns}$ of the initial NPT equilibration run and remained there for the remaining $0.8 \mathrm{~ns}$ of the NPT setup run as well as the full $7 \mathrm{~ns}$ of the subsequent NVT run.

\subsection{DA species have varying dif- fusivities on graphene.}

From these simulations, we calculated the diffusion constants of adatom(DA), DA, DAH ${ }^{+}$, $\mathrm{DOQ}$, and $\mathrm{DOQH}^{+}$on flat graphene. Figure 2 plots the directional mean squared displacements (MSDs) and velocity auto-correlation functions (VACFs) of these adsorbates, where 

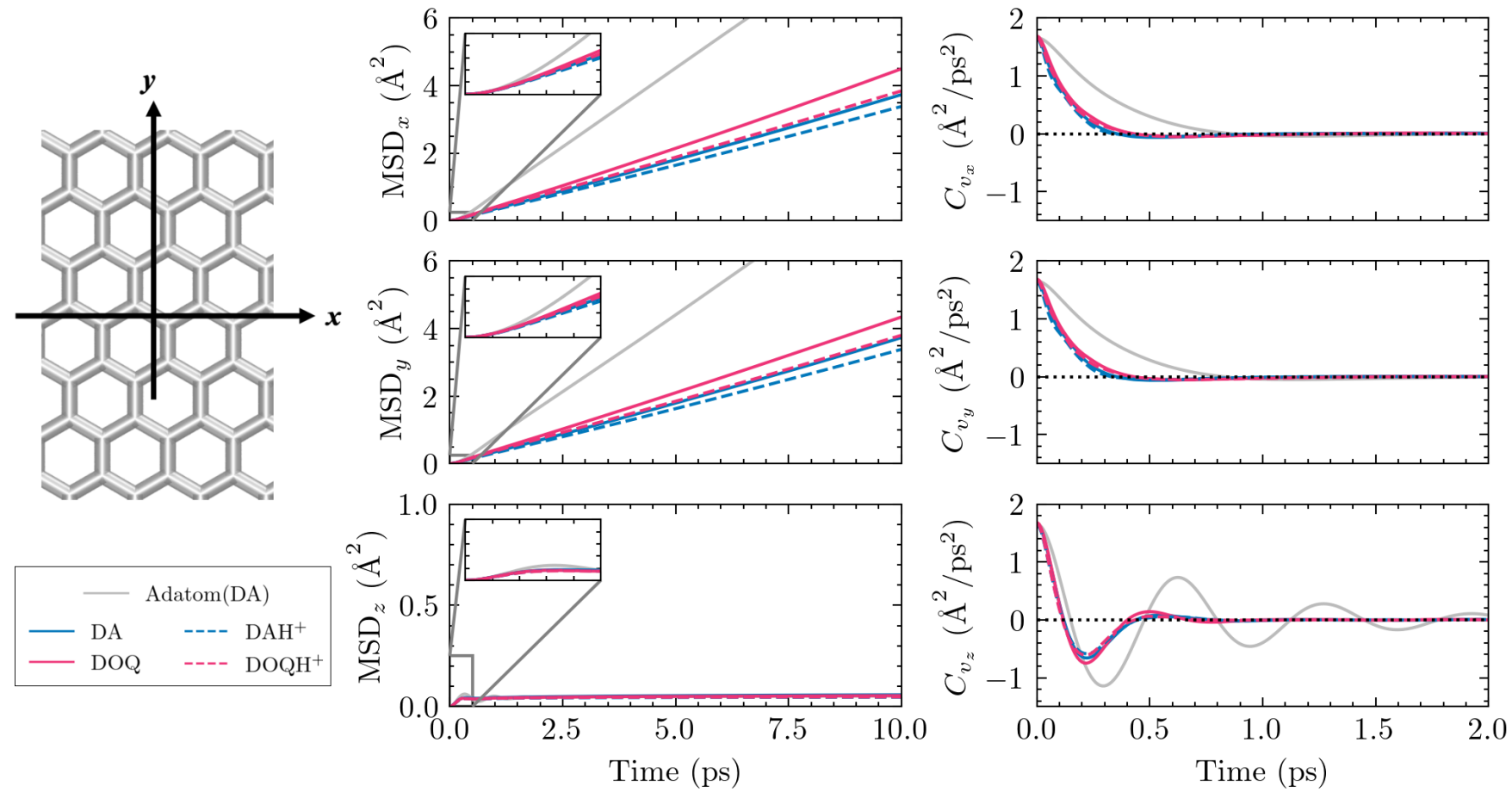

Figure 2: Mean squared displacements and velocity auto-correlations of DA species on graphene. Mean squared displacements (MSD, left column) and velocity auto-correlation functions, $\operatorname{VACFs}\left(C_{v}\right.$, right column) are shown as a function of time along three directions: $x$ and $y$ lie on the graphene plane (as shown in the schematic on the left), while $z$ is normal to the plane. The inset plots show the enlarged MSD curves in the 0-0.5 ps and 0-0.25 $\AA^{2}$ range. MSDs and VACFs were collected from ten 5 ps $N V T$ trajectories at $300 \mathrm{~K}$ at a recording interval of 0.01 ps. Plots showing the comparison of the MSDs for all ten trials of each DA species are shown in Figure S2.

Table 1: Diffusivities of DA species on graphene.

\begin{tabular}{c|ccc|c}
\hline Adsorbates & $D_{x}\left(\times 10^{-5} \mathrm{~cm}^{2} / \mathrm{s}\right)$ & $D_{y}\left(\times 10^{-5} \mathrm{~cm}^{2} / \mathrm{s}\right)$ & $D_{z}\left(\times 10^{-5} \mathrm{~cm}^{2} / \mathrm{s}\right)$ & $D\left(\times 10^{-5} \mathrm{~cm}^{2} / \mathrm{s}\right)^{(\mathrm{b})}$ \\
\hline Adatom(DA) & $4.59 \pm 0.32^{(\mathrm{c})}$ & $4.71 \pm 0.31$ & $0.00 \pm 0.01$ & $4.65 \pm 0.29$ \\
DA & $1.92 \pm 0.12$ & $1.92 \pm 0.13$ & $0.00 \pm 0.00$ & $1.92 \pm 0.07$ \\
DAH $^{+}$ & $1.73 \pm 0.13$ & $1.74 \pm 0.15$ & $0.00 \pm 0.00$ & $1.74 \pm 0.07$ \\
DOQ $^{\text {DOQH }}{ }^{+}$ & $2.34 \pm 0.22$ & $2.23 \pm 0.17$ & $0.00 \pm 0.00$ & $2.29 \pm 0.16$ \\
DA-in-water $^{(\mathrm{d})}$ & $1.96 \pm 0.24$ & $1.94 \pm 0.15$ & $0.00 \pm 0.00$ & $1.95 \pm 0.14$ \\
\hline
\end{tabular}

(a) Directional diffusion coefficients, $D_{x}, D_{y}$, and $D_{z}$, were computed by linearly fitting the MSD curves in Fig. 2 using the Einstein relation from 4-10 ps. (b) The 2D diffusion coefficient, $D$, was computed from the arithmetic mean of $D_{x}$ and $D_{y} .{ }^{\text {(c) }}$ All standard deviations are reported across ten independent $5 \mathrm{~ns} N V T$ trajectories at $300 \mathrm{~K}$. (d) The last row is the directional diffusion coefficients of DA in a cubic $(100 \AA)^{3}$ simulation box consisting only of water. (e) For "DA-in-water," $D$ is the $3 \mathrm{D}$ diffusion coefficient.

the $x$ and $y$ directions lie along the graphene plane, and the $z$ direction extends out from the surface. The linear region of the MSD plots were then used to calculate the directional diffusion coefficients reported in Table 1. As discussed in the Methods, it should be noted that the reported values of $D$ in Table 1 will be an overestimate of the $D_{\infty}$ values due to the finite size of the simulation box. The magnitude of the correction can be estimated by comparing the last two rows in Table $\mathrm{S} 2$, which indicates a value of $1.92 \times 10^{-5} \mathrm{~cm}^{2} / \mathrm{s}$ for $D_{\text {Graphene100, }}$, while $D_{\infty}$ is estimated to be $1.24 \times 10^{-5} \mathrm{~cm}^{2} / \mathrm{s}$. All $D$ values reported in the main text are those obtained directly from this largest simulation size, "Graphene100," which has graphene surface dimensions of $98.2 \times 97.8 \AA^{2}$. A more detailed discussion can be found in the SI.

For all species, both the displacement and velocity autocorrelations in the $z$ direction show 
evidence of the species' adsorption on the surface, with extremely limited displacements in the $\mathrm{MSD}_{z}$ and evidence of a region of anticorrelation in the corresponding $\mathrm{VACF}, C_{v_{z}}$, which together reflect the constrained motion in the $z$-direction. In contrast, the $\mathrm{MSD}_{x}$ and $\mathrm{MSD}_{y}$ plots show evidence of the initial inertial regime followed by diffusive behavior for all species with no significant regions of anticorrelation observed in either $C_{v_{x}}$ or $C_{v_{y}}$.

In order to evaluate the accuracy of DA dynamics within these MD simulations, it is helpful to first benchmark the diffusion constant obtained from our simulations of a fully solvated DA, $D=1.53 \times 10^{-5} \mathrm{~cm}^{2} / \mathrm{s}$ (see Table 1, last row), with that obtained from flow injection analysis experiments, $D=0.6 \times 10^{-5} \mathrm{~cm}^{2} / \mathrm{s}$. $\frac{45}{}$ The correspondence between these two values is impressive given the different ways they were obtained. We can then compare our simulation-derived diffusion coefficient for DA on the $98.2 \AA$ by $97.8 \AA$ graphene surface, $1.92 \times 10^{-5} \mathrm{~cm}^{2} / \mathrm{s}$, to that obtained for fully solvated DA in a cubic $(100 \AA)^{2}$ box, $1.53 \times 10^{-5}$ $\mathrm{cm}^{2} / \mathrm{s}$, which indicates that the overall diffusivity of adsorbed and solvated DA are quite similar, albeit the former is only free to diffuse on the two-dimensional surface.

The results in Figure 2 and Table 1 also showed that the structureless adatom adsorbate diffuses more rapidly than the molecular adsorbates. DA is significantly larger than the atomic adsorbate modeled here and asymmetric in both shape and charge distribution. For comparison, the adatoms are $2.64 \AA$ in diameter, which is slightly smaller than the dimension of the hexagonal lattice spacing of graphene, 2.84 $\AA$ across. Although the aromatic ring of DA is the same size as the lattice spacing, the diol and amine groups extend out from the ring in opposite directions, making its molecular structure larger than the lattice spacing. The larger and more extended structure also means that the molecular adsorbate will have additional collisions with the solvating water molecules, which we would expect to result in a more rapid velocity decorrelation, as is evident in $C_{v_{x}}$ and $C_{v_{y}}$ in Figure 2.

Among the DA-derived molecular species, small, but consistent, shifts are apparent in comparing the calculated diffusion constants for the protonated vs. deprotonated as well as the oxidized vs. reduced species. The protonated species have smaller diffusion constants: 1.74 vs. $1.92\left(\times 10^{-5} \mathrm{~cm}^{2} / \mathrm{s}\right)$ for $\mathrm{DAH}^{+}$vs. DA; and 1.95 vs. $2.29\left(\times 10^{-5} \mathrm{~cm}^{2} / \mathrm{s}\right)$ for $\mathrm{DOQH}^{+}$ vs. DOQ. A slightly larger shift can be seen in comparing the diffusion constants of the reduced vs. the oxidized species as well: 1.92 vs. $2.29\left(\times 10^{-5} \mathrm{~cm}^{2} / \mathrm{s}\right)$ for DA vs. DOQ; and 1.74 vs. $1.95\left(\times 10^{-5} \mathrm{~cm}^{2} / \mathrm{s}\right)$ for $\mathrm{DAH}^{+}$vs. $\mathrm{DOQH}^{+}$. These shifts hold between different DA species, and their consistency across different simulation runs can be seen clearly in Figure S2. We further investigate and discuss the origins of these shifts below.

\subsection{Molecular adsorbates show only slight preferences for different lateral positions on the graphene surface.}

Previous computational work has shown that the atomic-level structuring of graphene and its chirality in CNTs can influence the surface dynamics of an atomic adatom. ${ }^{49}$ In this section we compare the lateral distributions of adatom(DA), the center of mass (COM) of the molecular DA species, and the COM of their constituent chemical moieties in order to investigate the influence of the hexagonal lattice on adsorbate position. Figures 3 \& 4 display lateral distributions of the adsorbed species projected onto the graphene surface, for the COM of each species (Fig. 3) as well as the COM of various chemical groups within DA and DOQ (Fig. 4).

In Figure 3, the 2D COM distributions of adatom(DA) show a clear preference for the adatom to be located at the center of each hexagonal ring. However, no such preference is seen for the COM of the molecular DA species. Indeed, in Figure 4, only slight preferences can be seen for the phenyl ring and diol/quinone COM placements that correspond to a favorable slipped ring stacking configuration,,$\underline{62[63}$ and no lateral patterning is apparent for the location 

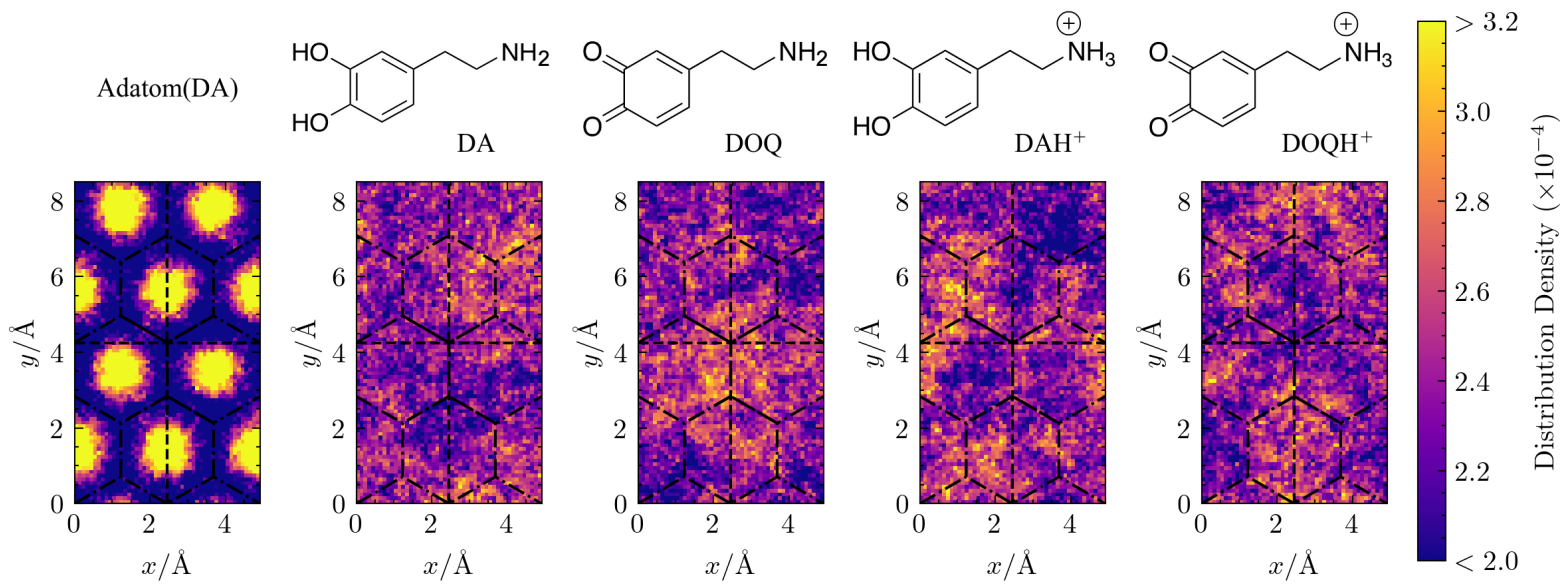

Figure 3: Lateral COM distributions of adatom(DA) and the molecular DA species on flat graphene. From left to right, the five subplots show results for adatom(DA), DA, DOQ, $\mathrm{DAH}^{+}$, and $\mathrm{DOQH}^{+}$, respectively. The projected COM coordinates on the surface were wrapped into 4 unit cells, which are separated by dashed lines. The dot-dashed lines represent the carbon-carbon bonds. Results were collected from ten $5 \mathrm{~ns} N V T$ trajectories at $300 \mathrm{~K}$ at a recording interval of $0.01 \mathrm{ps}$, and the colormaps show the distribution density on the carbon surface with a spatial resolution of $0.1 \times 0.1 \AA^{2}$.
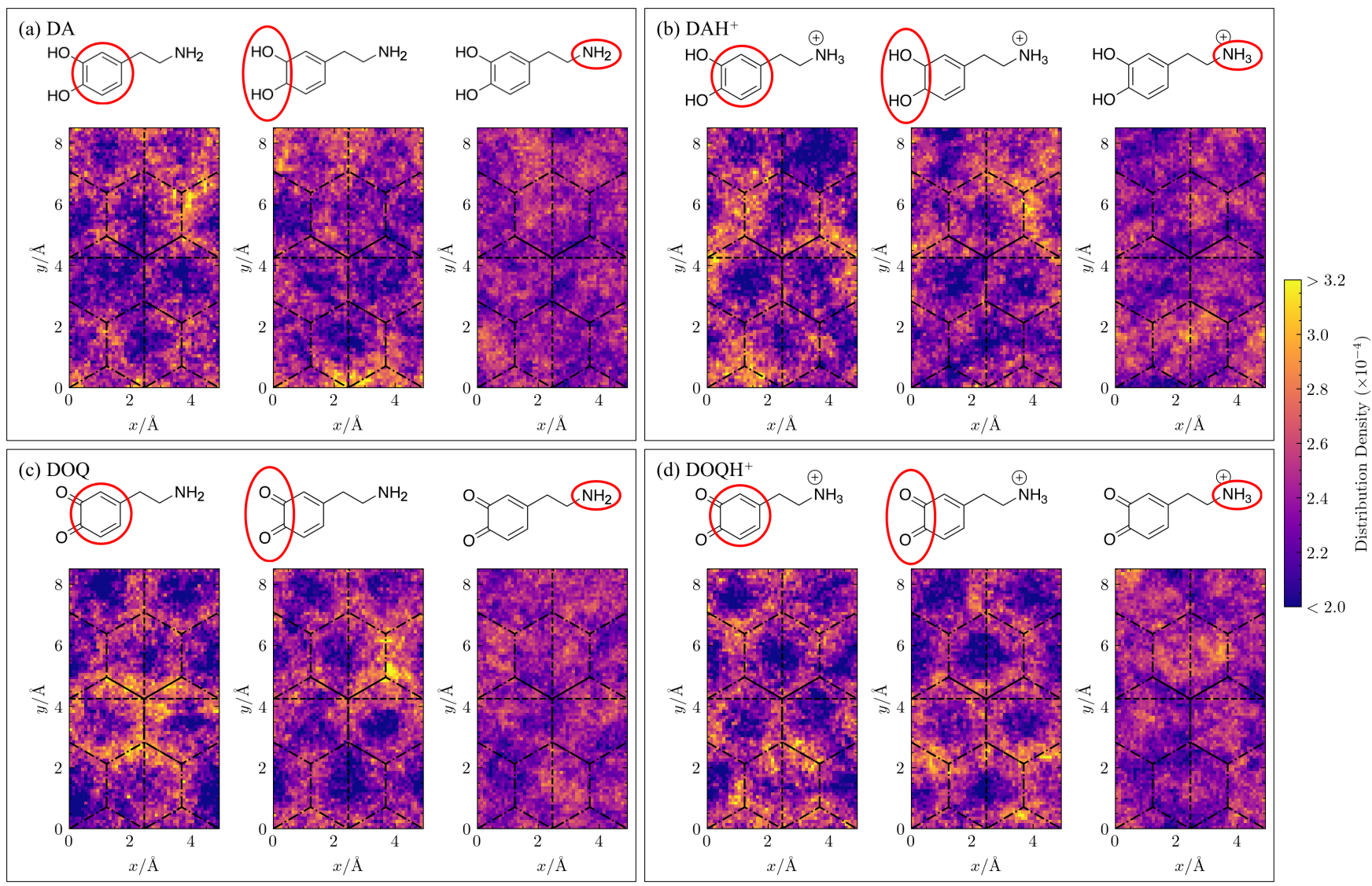

Figure 4: Lateral distributions of three functional groups of (a) DA, (b) $\mathbf{D A H}^{+}$, (c) DOQ, and (d) $\mathrm{DOQH}^{+}$, all on flat graphene. From left to right, the three plots show results for the phenyl ring, diol/quinone group, and amine group, respectively. The projected COM coordinates for each moiety on the surface were wrapped into 4 unit cells, which are separated by dashed lines. The dot-dashed lines represent the carbon-carbon bonds. Results were collected from ten $5 \mathrm{~ns} N V T$ trajectories at $300 \mathrm{~K}$ at a recording interval of $0.01 \mathrm{ps}$, and the colormaps show the distribution density on the carbon surface with a spatial resolution of $0.1 \times 0.1 \AA^{2}$. 
of the amine's COM.

\subsection{Vertical distributions of the amine groups depend on pro- tonation.}

Vertical distributions shown in Figure 5 characterize the spatial distribution of the adsorbate along the direction normal to the graphene surface, where the distance, $d$, is computed between the COM of a molecule or a chemical group and the closest point on the carbon surface. Considered alongside the lateral distributions, these vertical distributions help explain the differences in surface dynamics observed between the different DA species.

The vertical distributions of the phenyl ring and diol/quinone moieties are relatively narrow, located just above the graphene surface at about $3 \AA$, and remain nearly identical across all DA species. In contrast, the vertical distributions of the amine group are much broader, peak at about $6 \AA$ above the graphene surface, and display a significant dependence upon the amine protonation state (see Figure 5, rightmost panel). For the protonated species, the vertical distributions of the amine group shift further from the surface for both the oxidized and reduced species.

To better understand this shift, in Fig. 6] we focus on the peaks within these vertical amine distributions and show a series of representative $\mathrm{DA}$ and $\mathrm{DAH}^{+}$configurations at those distances. In Fig. 6a, the amine distance distributions of the charge-neutral species, DA and DOQ, are remarkably similar, as are those of the protonated species, $\mathrm{DAH}^{+}$and $\mathrm{DOQH}^{+}$. For both neutral species, there is a smaller peak at about $3.7 \AA(i)$, a shoulder at about $5.0 \AA$ (ii), and a larger peak at $5.9 \AA$ (iii). For both protonated species, however, there is no peak at $3.7 \AA$, the shoulder at $\sim 5.0 \AA$ is greatly reduced $(i v)$, and the last peak is even larger and further shifted from the surface at $6.1 \AA$ $(v)$. As can be seen in the corresponding configurations in Fig. 6r, the peak closest to the surface corresponds to a configuration where the amine group is approximately the same distance from the surface as the phenyl ring. The shoulders in $(i i)$ and $(i v)$ correspond to configurations where one bond in the carbon linker between the phenyl ring and the amine group has rotated away from the surface. Finally, the largest peaks at $(i i i)$ and $(v)$ represent configurations with the linker fully rotated out away from the surface into the solvating water layer. This last configuration is the most probable one for all four species, and the dominant configuration for the protonated DA species. In this configuration, the amine group extends into the bulk water where it can more readily form hydrogen bonds and, when protonated, its positive charge can interact more directly with the polar solvent.

\subsection{Differences in $D$ between DA species reflect differences in their solvent interactions.}

To understand the differences we observed in $D$ across various DA species adsorbed at the graphene-water interface, it is helpful to separately consider the influence of the graphene surface and the aqueous solvent.

Lateral distributions of the adsorbates and their chemical groups in Figures $3 \& 4$ show only a slight dependence on the underlying graphene structure. In addition, the trends that are present do not explain the observed trends in $D$ values. The lateral COM distributions reveal significant energetic barriers only for the adatom(DA), however, this species diffuses the most rapidly of all those simulated. Moreover, if the energetic barriers of the graphene surface were the dominant factor, DA might be expected to diffuse the most rapidly of the molecular adsorbates and $\mathrm{DOQH}^{+}$the most slowly, based upon the degree of ordering displayed in the four panels in Figure 4. Instead, these two species have similar $D$ values, while DOQ diffusion is the most rapid and $\mathrm{DAH}^{+}$is the slowest. Taken together, these observations make it clear that the differences in diffusion constants observed in our simulations cannot be attributed to the observed differences in interactions with the pristine graphene surface, leading us to consider in more detail the influence of the aqueous solvent. 


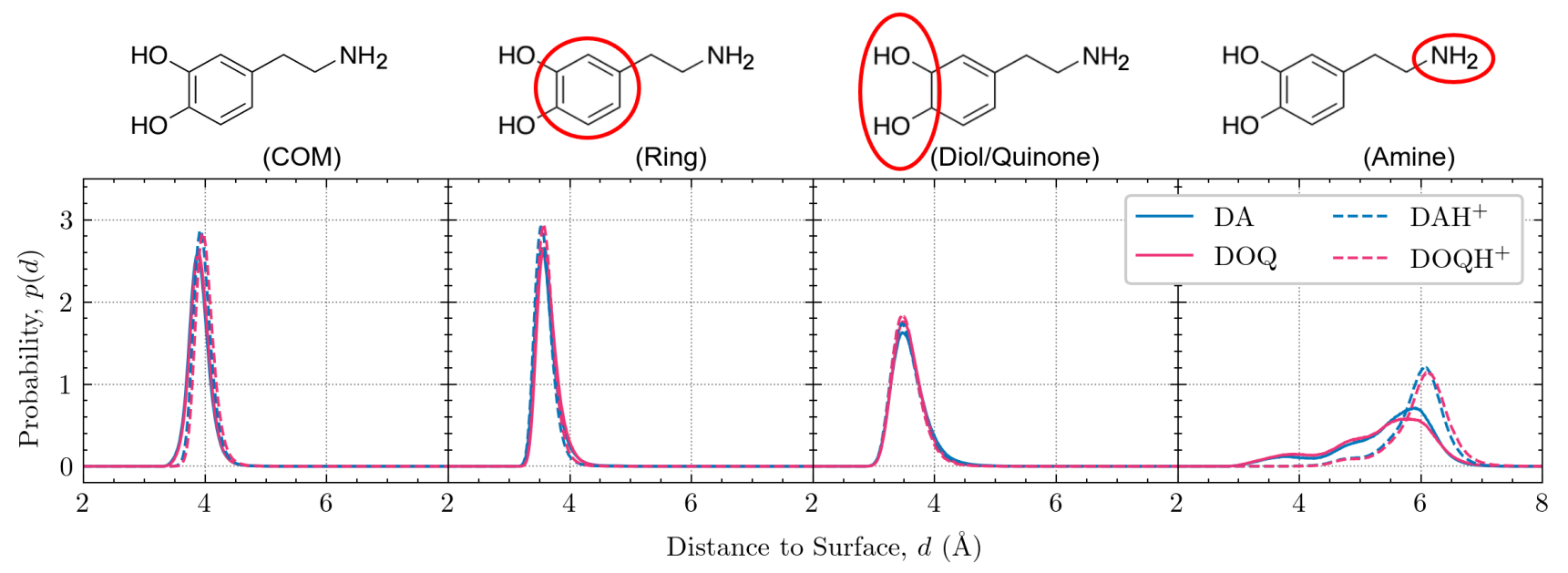

Figure 5: Vertical distributions of the center-of-mass (COM) and different moieties of DA and DOQ on the graphene surface. From left to right, the four plots show the vertical distributions of the adsorbate COM, its aromatic ring, its diol/quinone moiety, and its amine group, respectively. Colored curves within each subfigure indicate different adsorbates. $d$ is the distance to the surface, and $p(d)$ is its probability. Data for each distribution were collected from ten $5 \mathrm{~ns} N V T$ trajectories at $300 \mathrm{~K}$ at a recording interval of $0.01 \mathrm{ps}$.

To test the hypothesis that it is the solvent interactions that give rise to the observed differences in diffusivity, we simulated the diffusion of the neutral species, DA and DOQ, on a pristine graphene surface with no solvent. The results are shown in Figure 7 - the differences between the MSDs of DA and DOQ at the graphene-aqueous ("solvated") and graphenevacuum ("vacuum") interfaces are shown in Figure $7 \mathrm{a}$ for ten trials for each case, and a better view of the MSDs of the two species at the graphene-vacuum interface is shown in Figure $7 \mathrm{~b}$ for all forty trials of each species. As expected, the curves show that inertial dynamics dominates the motion of the DA species at the graphene-vacuum interface over a much longer time window than in the solvated case, which precludes the calculation of a diffusion constant in this time window. Moreover, considering the artificial lack of thermal fluctuations on the simulated graphene surface itself, the observed MSD curves cannot be taken as indicative of the realistic graphene-vacuum dynamics of these species. Nonetheless, this approach allows us to directly remove the influence of water within these simulations and enables us to observe two key differences between the dynamics of the adsorbates at the solvated and vacuum interfaces. First, the solvent is clearly responsible for a dramatic slow down in the diffusion of the adsorbed species. Second, the differences between DA and DOQ, which can be reliably seen in the segregation of the ten pink and ten blue traces for the solvated species in Figure $7 \mathrm{a}$, are not apparent in the species on the graphenevacuum interface in Figure $7 \mathrm{~b}$, where no similar segregation can be seen among the forty blue DA and forty pink DOQ traces. We therefore conclude that the difference between the $D$ values observed in our simulations of DA and DOQ arise from their interactions with the solvating water molecules.

The Stokes-Einstein equation $\sqrt[64]{67}$ describes how the diffusion constant, $D$, of a fully solvated spherical particle in non-turbulent flow depends on various properties of both the solvent and the diffusing particle:

$$
D=\frac{k_{\mathrm{B}} T}{c \pi \eta R_{\mathrm{H}}},
$$

where $c$ is a numerical constant depending on boundary conditions at the solvent-particle interface, $k_{\mathrm{B}}$ is the Boltzmann constant, $T$ is the temperature, $\eta$ is the solvent viscosity, and $R_{\mathrm{H}}$ is the hydrodynamic radius of the diffusing particle. 646869

The direct applicability of the Stokes-Einstein (SE) equation is limited in this case since the adsorbates are not fully solvated. In addition, DA is not spherical and only moderately larger 


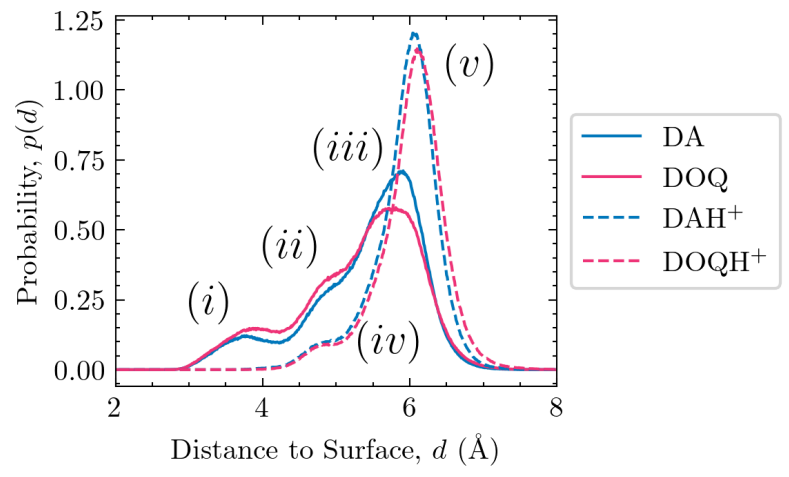

(a)

\begin{tabular}{c|cc}
\hline \multirow{2}{*}{ Peaks } & \multicolumn{2}{|c}{$d(\AA)$} \\
\cline { 2 - 3 } & $\mathrm{DA}$ & $\mathrm{DOQ}$ \\
\hline (i) & 3.68 & 3.77 \\
(ii) & 5.22 & 4.94 \\
(iii) & 5.91 & 5.84 \\
\hline \multirow{3}{*}{ (iv) } & $\mathrm{DAH}^{+}$ & $\mathrm{DOQH}^{+}$ \\
\cline { 2 - 3 }$(v)$ & 4.76 & 4.94 \\
(v) & 6.09 & 6.12 \\
\hline
\end{tabular}

(b) $(i)$

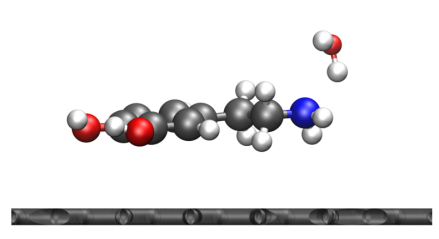

(ii)

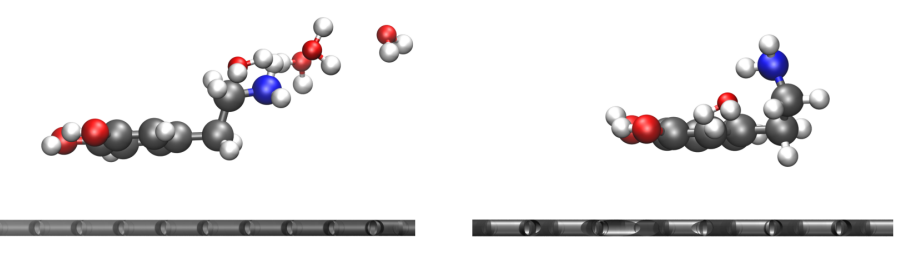

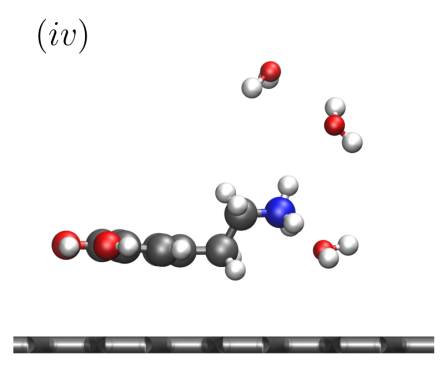

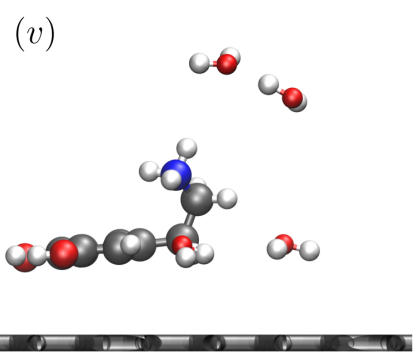

(c)

Figure 6: Amine group distributions and configurations. (a) Vertical distributions of the amine groups from Fig. 5 are shown here in more detail for $\mathrm{DA}, \mathrm{DOQ}, \mathrm{DAH}^{+}$, and $\mathrm{DOQH}^{+}$. Peaks in these distributions are labeled and correspond to the vertical distances and conformations shown in (b) and (c). In (b), the positions are listed for the peaks of the vertical distance distributions, which were obtained from curve-fitting using Gaussian functions. In (c), representative configurations of the adsorbate and the amine-solvating waters (within $5 \AA$ of the amine $\mathrm{N}$ ) are shown for each identified distance position for DA $(i-i i i)$ and $\mathrm{DAH}^{+}(i v, v)$.

than the solvating water molecules - both of which can give rise to deviations from the SEdescribed behavior ${ }^{70}$ Finally, significant interactions between the solvent and the solute are expected to yield an effective hydrodynamic radius larger than the actual particle radius, the size of which depends on the strength of those interactions. 68 Since changes in asphericity and size are negligible between the different DA species, we focus here on the expected variations in the effective $R_{\mathrm{H}}$ to determine if they can explain the differences in diffusivity observed in the simulations between the neutral and protonated species and between the oxidized and reduced species.

First we consider the observation that the charged species, $\mathrm{DAH}^{+}$and $\mathrm{DOQH}^{+}$, both display a lower $D$ value in our simulations than their uncharged counterparts, DA and DOQ (see Table 1). The positively-charged $\mathrm{DAH}^{+}$ and $\mathrm{DOQH}^{+}$have stronger electrostatic interactions with water than the neutral DA and DOQ species, which will increase their effective hydrodynamic radii and correspondingly decrease their diffusivities, $D$. Indeed, simulations have shown that the effective hydrodynamic radius of a small nanoparticle directly depends upon its charge. ${ }^{65}$ In addition, the shift 
(a)

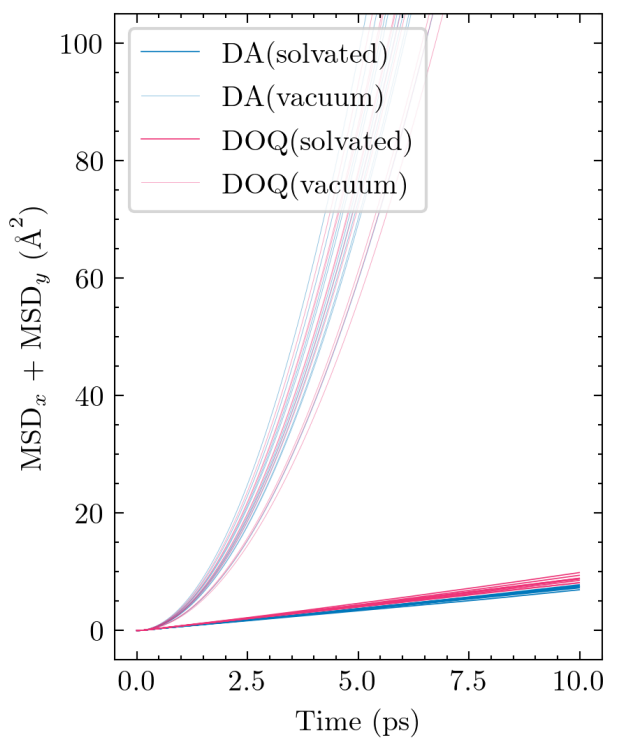

(b)

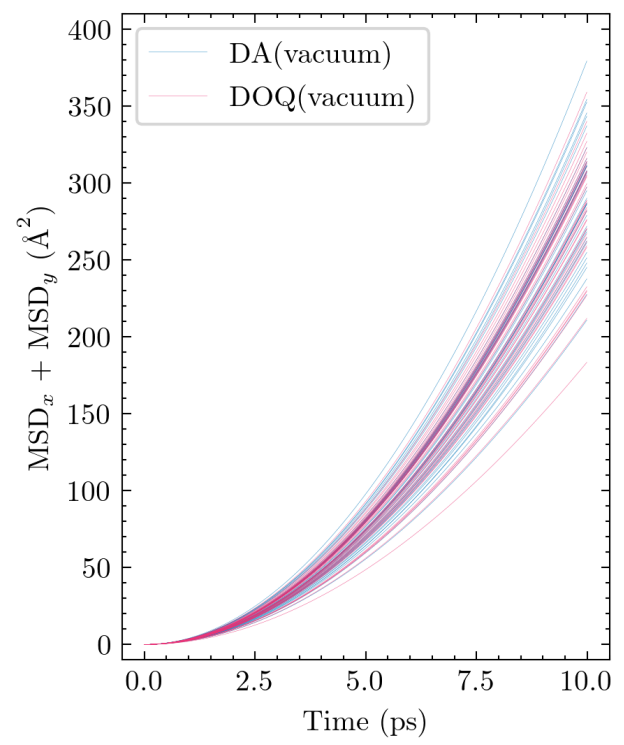

Figure 7: In-plane mean squared displacements of DA and DOQ at the aqueous-graphene and vacuumgraphene interfaces. (a) The comparison between DA (blue) and DOQ (pink) at the two interfaces is shown for ten independent 5 ps $N V E$ vacuum simulations (lighter colors), and ten independent 5 ps $N V T$ solvated simulations at $300 \mathrm{~K}$ (darker colors), both at a recording interval of 0.01 ps. (b) The MSDs for DA (blue) and DOQ (pink) are shown from forty independent trajectories each for the $N V E$ vacuum simulations. Please note the different scales of the MSD axes in the two plots.

in the amine position towards the solvent in the protonated species (see Figure 6) clearly reflects its increased attractive interactions with the solvent. Moreover, this amine shift enhances the effect of its additional charge on the effective hydrodynamic radius by enabling it to interact with the solvent in a manner more closely mimicking that of a fully solvated molecule. We therefore conclude that the observed decrease in the diffusivities of DA and DOQ upon protonation results from increased interactions with the solvent, due to both the additional electrostatic interactions between the polar solvent and the charged species as well as the corresponding shift towards more solvated configurations for the protonated amine.

Second, we consider the observed trend that the oxidized species, DOQ and $\mathrm{DOQH}^{+}$, both display a larger $D$ value in our simulations than their reduced counterparts, DA and $\mathrm{DAH}^{+}$(see Table 1). The difference between these species lies in the oxidation of the two alcohol groups to form a quinone group. Experimental studies have shown that the diffusion of a water molecule is significantly slowed when it moves through a solvent capable of forming hydrogenbonds. $\frac{73}{7 m}$ Importantly, the alcohols in DA and
$\mathrm{DAH}^{+}$are able to participate in hydrogen bonding with the solvating water molecules as both hydrogen bond donors and hydrogen bond acceptors, while the ketones that constitute the quinone moiety in $\mathrm{DOQ}$ and $\mathrm{DOQH}^{+}$are only able to act as hydrogen bond acceptors and can thus form fewer hydrogen bonds with the solvent. Despite this difference, the position of the diol/quinone moiety does not shift vertically upon oxidation, which reflects the strength of the favorable attractions between the phenyl ring and the graphene surface. However, the diol/quinone moieties still interact with solvating water molecules, and the additional hydrogen bonding possible for the alcohol groups in the DA and $\mathrm{DAH}^{+}$species should increase their effective hydrodynamic radii, in comparison to those of $\mathrm{DOQ}$ and $\mathrm{DOQH}{ }^{+}$, and thereby reduce their $D$ values, which is precisely the trend observed in the simulations.

To summarize, given the complexities of the aqueous-graphene interface and the solutesolvent interactions, the Stokes-Einstein equation cannot be used to predict the precise diffusion constants for these molecular DA species. However, the SE relation between diffusivity and the effective hydrodynamic radius explains 
both the observed trends in $D$, demonstrating the importance of the solvent in determining the dynamics of these DA species on the microelectrode surface.

\section{Discussion}

The simulations in this study provide novel atomistic-level detail on the structure and dynamics of various dopamine species adsorbed onto a pristine graphene microelectrode surface. These details yield unique insight regarding the necessary components for dopamine adsorption to the microelectrode surface, details on diffusion timescales and determinants, and implications for various experimental conditions. The main conclusion is that dopamine and its oxidation product DOQ have fast diffusion on a graphene surface, and thus they should be able to sample many domains on a microelectrode surface.

Adsorbed dopamine stability and structure. First, we find that DA, DOQ, and their protonated counterparts all readily adsorb from the aqueous phase onto a pristine, flat graphene surface. For all species, adsorption occurred rapidly, and not a single desorption event was observed over the ns timescale of our equilibrium MD simulations. Moreover, since the OPLS-AA force field employed in this work is expected to slightly underestimate the attractions acting between the adsorbate and the graphene surface, ${ }^{59}$ adsorption is expected to be even more robust in the physical system. Although the standard FSCV holding potential of $-0.4 \mathrm{~V}$ was designed to maximize DA adsorption $^{23}$ and surface oxidation is known to enhance adsorption, 29130 it is important to note that we find neither an applied potential nor surface oxidation necessary for a single DA to adsorb to and remain on the basal plane of pristine graphene. This conclusion is supported by various experimental studies that point to DA's ability to adsorb directly to pristine graphene surfaces, from early studies on freshly fractured graphene $^{\sqrt{30}}$ to more recent work on HOPG surfaces. 33

Second, the vertical distributions shown in Figure $6 a$ and Figure $6 c(v)$, indicate that the protonated amines in our simulations (which have no applied potential) extend out into the solvent at nearly all times and therefore cannot be a primary contributor to the favorable energetic interactions driving the robust surface adsorption we observed for $\mathrm{DAH}^{+}$and $\mathrm{DOQH}^{+}$. While early experimental work appeared to show that amine groups were necessary for adsorption, 24 our conclusions here are supported by later work by Allred and coworkers demonstrating the ready adsorption of catechols, even those lacking an amine group, to freshly fractured glassy carbon surfaces. $\stackrel{30}{ }$ We note, however, that the same study still observed an increase in the adsorption of $\mathrm{DAH}^{+}$, with its positively-charged amine, to oxidized carbon microelectrode surfaces, $\stackrel{30}{ }$ as have subsequent experiments. 29174755 Interestingly, the sensitivity of the amine configuration to its charge state, as observed in these simulations, suggests that DA's interactions with the graphene surface and the solvating waters will shift significantly during a CV experiment as the applied potential scans over a range of voltages; differences in DA's configuration at each voltage will play an important role in determining the shifting equilibrium constant between adsorbed and desorbed DA species during a cyclic scan.

Dopamine diffusion dynamics. We have also gained new insight into the dynamics of these dopamine species in bulk solvent as well as on the pristine graphene surface, both at the aqueous and vacuum interfaces. To start, we note again the similarity between the diffusion constants we calculate here for DA in bulk water $\left(1.53 \times 10^{-5} \mathrm{~cm}^{2} / \mathrm{s}\right)$ and on the graphene surface $\left(1.92 \times 10^{-5} \mathrm{~cm}^{2} / \mathrm{s}\right)$. Given the experimentally-observed adsorption-related lag time for DA signal detection,, $29[43$ this similarity suggests that adsorption and desorption are occurring at a slower rate than diffusion on the electrode surface.

Importantly, simulations were performed both in the presence of explicit solvating waters as well as in a vacuum for comparison purposes, enabling us to determine the effect of the aqueous interface on analyte dynamics. First, we note that the diffusion of DA and DOQ at the graphene-vacuum interface is far faster than at 
the graphene-water interface (Fig. $7 \mathrm{a}$ ), and the difference between DA and DOQ diffusion at the graphene-water interface disappears at the graphene-vacuum interface (Fig. 7b). These results demonstrate the essential role that solvating waters play in determining the adsorbate's motion on pristine graphene. Indeed, the differences in diffusivities between the protonated and deprotonated and the oxidized and reduced species at the graphene-water interface can be fully explained by differences in their effective hydrodynamic radii in the solvating water phase.

These observations lead us to three conclusions regarding the determinants of analyte motion on the graphene microelectrode surface. First, small changes in an analyte's chemical composition can easily influence its surface diffusivity by altering its effective hydrodynamic radius in the solvating phase - even if the changes do not appreciably influence its interactions with the graphene surface itself. Second, slight changes to the internal structure of a diffusing analyte - for instance, when an applied potential pulls particular moieties closer to the electrode surface during a portion of a cyclic scan - may also influence its surface diffusivity, either solely through changes in analyte-solvent interactions or more directly through shifts in the analyte-electrode interactions. In the case of dopamine, the protonated amines we simulated preferred to extend out towards bulk water and thereby interact with more solvating water molecules. However, under a negative holding potential, the protonated amine would be attracted to the surface and thus unable to participate in some portion of these water interactions. As a result, the holding potential could either increase or decrease $\mathrm{DAH}^{+}$'s surface diffusivity, depending on the relative balance between an increase in the diffusion rate, from the expected decrease in the effective hydrodynamic radius, and a decrease in the diffusion rate rate, from the expected increase in the analyte-surface interactions. Third, we conclude that analyte surface dynamics will be sensitive to changes in the dynamics of the solvating water. Results from multiple studies suggest that the mobility of water under nanoscale confinement depends sensitively on the geometry of the confining regions and the geometry-dependent energy landscape experienced by water at the surface. ${ }^{\sqrt{76}}$ Recent innovations in carbon microelectrode architecture that involve the introduction of nanoscale patterning or pores will clearly influence the dynamics of the solvating water. It is already recognized that the diffusion dynamics of the analyte towards and away from the electrode will be influenced by these geometric constraints, and this has been seen in the thin layer effects observed for electrode surfaces with micron scale roughness. 15$] 36+38$

An investigation of the influence of additional functional groups or other defects on the graphene surface lies outside the scope of this initial work. However, we note that any sitespecific surface interactions with the analyte, for instance between a positively charged amine group on DA and a graphitic oxide species, is expected to significantly impact its surface dynamics or even arrest its motion for lengths of time. The presence of multiple adsorbates of the analyte, its oxidized derivative, or other contaminating organic material - may also significantly complicate and alter diffusion dynamics. Future studies will be needed to address these questions.

\section{Implications for Experimental Timescales.} The simulation-derived diffusivities of various DA species at the pristine graphene-water interface provide the information needed to translate observed FSCV scan-rate dependencies to length-scales over which a DA analyte can be expected to diffuse during a single scan. Evidence has long suggested that certain domains on carbon fiber electrode surfaces have an increased electrochemical activity ${ }^{47 / 81}$ Even pristine graphene surfaces have recently been shown to display spatially heterogeneous electrode activity. ${ }^{82}$ In one particularly interesting study, Oleinick and coworkers identified ranges of scan rates between which DA detection varies considerably. $\stackrel{47}{ }$ They concluded that at the lowest scan rates $(\leq 100 \mathrm{~V} / \mathrm{s})$, all adsorbed DA are able to diffuse to electrochemically active domains for oxidation, while at the highest scan rates $(\geq 1000 \mathrm{~V} / \mathrm{s})$, only adsorbed DA within 
an active domain would be oxidized and detected. If we take the value of $D=1.73 \times 10^{-5}$ $\mathrm{cm}^{2} / \mathrm{s}$ for $\mathrm{DAH}^{+}$on pristine graphene, as calculated in our simulations, and use it to estimate the root mean squared distance traveled by $\mathrm{DAH}^{+}$during a single oxidation scan of FSCV, these timescales map to estimated diffusion distances of $\leq 3.4 \mu \mathrm{m}$ for the fastest scans and $\geq 10.8 \mu \mathrm{m}$ for the slower scans. For a typical scan rate of $400 \mathrm{~V} / \mathrm{s}$, this distance corresponds to $5.4 \mu \mathrm{m}$. Notably, for slower scans, the diffusion distance is on the order of the diameter of a typical carbon-fiber electrode. Of course, this estimate assumes that diffusion is occurring across a pristine and homogeneous graphene surface, which neglects entirely the differences in diffusivities that would be expected between the regions of differing electrochemical activities. Nonetheless, it provides a baseline estimate for how far we might expect $\mathrm{DAH}^{+}$to diffuse during an experiment, as well as the magnitude of the difference expected between diffusion during a fast and a slow scan.

These experiments are the first to look directly at the diffusion of dopamine, DOQ, and their protonated species on graphene. The results show extremely fast diffusion on the surface, on the order of solution diffusion rates. Thus, $\mathrm{DAH}^{+}$and $\mathrm{DOQH}^{+}$will sample much of the surface as they diffuse on a typical microelectrode. This insight is the first step in understanding dopamine-microelectrode interactions, which will enable the development of better electrode surfaces. Future studies will look at the effects of surface functional groups and applied voltage, and atomistic simulations will prove to be an important tool to predict surface behavior and thus electrochemistry at electrodes.

\section{Conclusions}

Carbon microelectrode surface structure can dramatically influence the time-resolution and signal-to-noise ratio of electroanalytical measurements, as well as impact their sensitivity and specificity. ${ }^{15535}$ However, atomistic simulations have not yet been employed to probe how the transport of molecular neurotransmitters on these carbon surfaces depend upon their structural features. In this work, we simulated the diffusion of DA, DOQ, and their protonated counterparts on the simplest of these surfaces - the pristine basal plane of graphene. We found that adsorbate motion was highly dependent upon interactions between the adsorbates and the solvating liquid water, which gave rise to differences in the diffusivities between the protonated and deprotonated and between the oxidized and reduced species. This investigation has advanced our understanding of the diffusion-controlled surface transport for dopamine on the pristine graphene surface and has laid the groundwork for future investigations to determine the influence of more complex microelectrode surface structures on analyte dynamics. Such studies will be essential for using atomistic simulations to predict dopamine surface diffusivity and how it influences electrochemistry.

Acknowledgement This work was supported by an NIH grant, NIH R01EB026497, to B.J.V. Additional support was provided by funding from the University of Virginia. The authors also acknowledge Research Computing at the University of Virginia (https://rc.virginia.edu) for providing computational resources and technical support.

\section{References}

(1) Song, Y.; Xu, C.; Kuroki, H.; Liao, Y.; Tsunoda, M. Recent trends in analytical methods for the determination of amino acids in biological samples. Journal of Pharmaceutical and Biomedical Analysis 2018, 147, 35-49.

(2) Shin, M.; Wang, Y.; Borgus, J. R.; Venton, B. J. Electrochemistry at the Synapse. Annual Review of Analytical Chemistry 2019, 12, 297-321.

(3) Puthongkham, P.; Venton, B. J. Recent advances in fast-scan cyclic voltammetry. Analyst 2020, 145, 1087-1102. 
(4) Sanghavi, B. J.; Wolfbeis, O. S.; Hirsch, T.; Swami, N. S. Nanomaterialbased electrochemical sensing of neurological drugs and neurotransmitters. Microchimica Acta 2015, 182, 1-41.

(5) Musameh, M. M.; Dunn, C. J.; Uddin, M. H.; Sutherland, T. D.; Rapson, T. D. Silk provides a new avenue for third generation biosensors: Sensitive, selective and stable electrochemical detection of nitric oxide. Biosensors and Bioelectronics 2018, 103, 26-31.

(6) Qian, Q.; Hu, Q.; Li, L.; Shi, P.; Zhou, J.; Kong, J.; Zhang, X.; Sun, G.; Huang, W. Sensitive fiber microelectrode made of nickel hydroxide nanosheets embedded in highly-aligned carbon nanotube scaffold for nonenzymatic glucose determination. Sensors and Actuators B: Chemical 2018, 257, 23-28.

(7) Ju, J.; Chen, W. In Situ Growth of Surfactant-Free Gold Nanoparticles on Nitrogen-Doped Graphene Quantum Dots for Electrochemical Detection of Hydrogen Peroxide in Biological Environments. Analytical Chemistry 2015, 87, 19031910.

(8) Sun, Y.; He, K.; Zhang, Z.; Zhou, A.; Duan, H. Real-time electrochemical detection of hydrogen peroxide secretion in live cells by $\mathrm{Pt}$ nanoparticles decorated graphene-carbon nanotube hybrid paper electrode. Biosensors and Bioelectronics 2015, 68, 358-364.

(9) Sanghavi, B. J.; Moore, J. A.; Chávez, J. L.; Hagen, J. A.; KelleyLoughnane, N.; Chou, C.-F.; Swami, N. S. Aptamer-functionalized nanoparticles for surface immobilization-free electrochemical detection of cortisol in a microfluidic device. Biosensors and Bioelectronics 2016, 78, 244-252.

(10) Feng, X.; Zhang, Y.; Zhou, J.; Li, Y.; Chen, S.; Zhang, L.; Ma, Y.; Wang, L.;
Yan, X. Three-dimensional nitrogendoped graphene as an ultrasensitive electrochemical sensor for the detection of dopamine. Nanoscale 2015, 7, 2427-2432.

(11) Salamon, J.; Sathishkumar, Y.; Ramachandran, K.; Lee, Y. S.; Yoo, D. J.; Kim, A. R.; kumar, G. G. One-pot synthesis of magnetite nanorods/graphene composites and its catalytic activity toward electrochemical detection of dopamine. Biosensors and Bioelectronics 2015, 64, 269-276.

(12) Taylor, I. M.; Robbins, E. M.; Catt, K. A.; Cody, P. A.; Happe, C. L.; Cui, X. T. Enhanced dopamine detection sensitivity by PEDOT/graphene oxide coating on in vivo carbon fiber electrodes. Biosensors and Bioelectronics 2017, 89, 400-410.

(13) Yang, C.; Denno, M. E.; Pyakurel, P.; Venton, B. J. Recent trends in carbon nanomaterial-based electrochemical sensors for biomolecules: A review. Anal. Chim. Acta 2015, 887, 17-37.

(14) Rodeberg, N. T.; Sandberg, S. G.; Johnson, J. A.; Phillips, P. E. M.; Wightman, R. M. Hitchhiker's Guide to Voltammetry: Acute and Chronic Electrodes for in Vivo Fast-Scan Cyclic Voltammetry. ACS Chemical Neuroscience 2017, 8, 221234.

(15) Yang, C.; Trikantzopoulos, E.; Nguyen, M. D.; Jacobs, C. B.; Wang, Y.; Mahjouri-Samani, M.; Ivanov, I. N.; Venton, B. J. Laser Treated Carbon Nanotube Yarn Microelectrodes for Rapid and Sensitive Detection of Dopamine in Vivo. ACS Sensors 2016, 1, 508-515.

(16) Schultz, W. Neuronal Reward and Decision Signals: From Theories to Data. Physiological Reviews 2015, 95, 853-951.

(17) Berridge, K. C.; Kringelbach, M. L. Pleasure Systems in the Brain. Neuron 2015, 86, 646-664. 
(18) Wightman, R. M. Probing Cellular Chemistry in Biological Systems with Microelectrodes. Science 2006, 311, 1570-1574.

(19) Gonon, F.; Buda, M.; Cespuglio, R.; Jouvet, M.; Pujol, J.-F. In vivo electrochemical detection of catechols in the neostriatum of anaesthetized rats: dopamine or DOPAC? Nature 1980, 286, 902-904.

(20) Bond, A. M. Past, present and future contributions of microelectrodes to analytical studies employing voltammetric detection. A review. Analyst 1994, 119, 1R-21R.

(21) Trojanowicz, M. Analytical applications of carbon nanotubes: a review. TrAC Trends in Analytical Chemistry 2006, 25, $480-$ 489.

(22) Zhang, Y.; Xiao, J.; Sun, Y.; Wang, L.; Dong, X.; Ren, J.; He, W.; Xiao, F. Flexible nanohybrid microelectrode based on carbon fiber wrapped by gold nanoparticles decorated nitrogen doped carbon nanotube arrays: In situ electrochemical detection in live cancer cells. Biosensors and Bioelectronics 2018, 100, 453-461.

(23) Venton, B. J.; Cao, Q. Fundamentals of fast-scan cyclic voltammetry for dopamine detection. Analyst 2020, 145, 1158-1168.

(24) Baur, J. E.; Kristensen, E. W.; May, L. J.; Wiedemann, D. J.; Wightman, R. M. Fast-scan voltammetry of biogenic amines. Analytical chemistry 1988, 60, 12681272 .

(25) Huffman, M. L.; Venton, B. J. Carbonfiber microelectrodes for in vivo applications. Analyst 2009, 134, 18-24.

(26) Kawagoe, K. T.; Zimmerman, J. B.; Wightman, R. M. Principles of voltammetry and microelectrode surface states. Journal of neuroscience methods 1993, 48, 225-240.

(27) Chen, P.; Fryling, M. A.; McCreery, R. L. Electron transfer kinetics at modified carbon electrode surfaces: the role of specific surface sites. Analytical Chemistry 1995, 67, 3115-3122.

(28) Jiang, Y.; Yang, L.; Sun, T.; Zhao, J.; Lyu, Z.; Zhuo, O.; Wang, X.; Wu, Q.; Ma, J.; Hu, Z. Significant Contribution of Intrinsic Carbon Defects to Oxygen Reduction Activity. ACS Catalysis 2015, 5, 6707-6712.

(29) Bath, B. D.; Michael, D. J.; Trafton, B. J.; Joseph, J. D.; Runnels, P. L.; Wightman, R. M. Subsecond adsorption and desorption of dopamine at carbon-fiber microelectrodes. Analytical chemistry 2000, 72, 5994-6002.

(30) Allred, C. D.; McCreery, R. L. Adsorption of catechols on fractured glassy carbon electrode surfaces. Analytical Chemistry 1992, 64, 444-448.

(31) Kneten, K. R.; McCreery, R. L. Effects of redox system structure on electrontransfer kinetics at ordered graphite and glassy carbon electrodes. Analytical Chemistry 1992, 64, 2518-2524.

(32) Dai, L. Carbon-based catalysts for metalfree electrocatalysis. Current Opinion in Electrochemistry 2017, 4, 18-25.

(33) Unwin, P. R.; Guell, A. G.; Zhang, G. Nanoscale electrochemistry of $\mathrm{sp}^{2}$ carbon materials: from graphite and graphene to carbon nanotubes. Accounts of chemical research 2016, 49, 2041-2048.

(34) Martin, A.; Hernandez-Ferrer, J.; Vazquez, L.; Martinez, M.-T.; Escarpa, A. Controlled chemistry of tailored graphene nanoribbons for electrochemistry: a rational approach to optimizing molecule detection. RSC Adv. 2014, 4, 132-139.

(35) Jacobs, C. B.; Ivanov, I. N.; Nguyen, M. D.; Zestos, A. G.; Venton, B. J. High Temporal Resolution Measurements of Dopamine with Carbon Nanotube Yarn Microelectrodes. 
Analytical Chemistry 2014, 86, 57215727.

(36) Cao, Q.; Hensley, D. K.; Lavrik, N. V.; Venton, B. J. Carbon nanospikes have better electrochemical properties than carbon nanotubes due to greater surface roughness and defect sites. Carbon 2019, 155, 250-257.

(37) Yang, C.; Hu, K.; Wang, D.; Zubi, Y.; Lee, S. T.; Puthongkham, P.; Mirkin, M. V.; Venton, B. J. Cavity carbon-nanopipette electrodes for dopamine detection. Analytical chemistry 2019, 91, 4618-4624.

(38) Shao, Z.; Puthongkham, P.; Hu, K.; Jia, R.; Mirkin, M. V.; Venton, B. J. Thin layer cell behavior of CNT yarn and cavity carbon nanopipette electrodes: Effect on catecholamine detection. Electrochimica Acta 2020, 361, 137032.

(39) Valentini, F.; Ciambella, E.; Conte, V.; Sabatini, L.; Ditaranto, N.; Cataldo, F.; Palleschi, G.; Bonchio, M.; Giacalone, F.; Syrgiannis, Z. et al. Highly selective detection of Epinephrine at oxidized SingleWall Carbon Nanohorns modified Screen Printed Electrodes (SPEs). Biosensors and Bioelectronics 2014, 59, 94-98.

(40) Oakes, L.; Westover, A.; MahjouriSamani, M.; Chatterjee, S.; Puretzky, A. A.; Rouleau, C.; Geohegan, D. B.; Pint, C. L. Uniform, Homogenous Coatings of Carbon Nanohorns on Arbitrary Substrates from Common Solvents. ACS Applied Materials \& Interfaces 2013, 5, 13153-13160.

(41) Puthongkham, P.; Yang, C.; Venton, B. J. Carbon Nanohorn-modified Carbon Fiber Microelectrodes for Dopamine Detection. Electroanalysis 2018, 30, 1073-1081.

(42) Puthongkham, P.; Venton, B. J. Nanodiamond coating improves the sensitivity and antifouling properties of carbon fiber microelectrodes. ACS sensors 2019, 4, 24032411.
(43) Venton, B. J.; Troyer, K. P.; Wightman, R. M. Response Times of Carbon Fiber Microelectrodes to Dynamic Changes in Catecholamine Concentration. Analytical Chemistry 2002, 74, 539-546.

(44) Bath, B. D.; Martin, H. B.; Wightman, R. M.; Anderson, M. R. Dopamine Adsorption at Surface Modified CarbonFiber Electrodes. Langmuir 2001, 17, 7032-7039.

(45) Gerhardt, G.; Adams, R. N. Determination of diffusion coefficients by flow injection analysis. Analytical chemistry 1982, 54, 2618-2620.

(46) Yan, L.; Chen, H.; Jing, C. TiO2 Facets Shaped by Concentration-Dependent Surface Diffusion of Dopamine. The journal of physical chemistry letters $\mathbf{2 0 1 9}, 10,898$ 903.

(47) Oleinick, A.; Álvarez Martos, I.; Svir, I.; Ferapontova, E. E.; Amatore, C. Surface Heterogeneities Matter in Fast Scan Cyclic Voltammetry Investigations of Catecholamines in Brain with Carbon Microelectrodes of High-Aspect Ratio: Dopamine Oxidation at Conical Carbon Microelectrodes. Journal of The Electrochemical Society 2018, 165, G3057-G3065.

(48) Zhang, H.-p.; Lin, X.-y.; Lu, X.; Wang, Z.; Fang, L.; Tang, Y. Understanding the interfacial interactions between dopamine and different graphenes for biomedical materials. Materials Chemistry Frontiers 2017, 1, 1156-1164.

(49) Shu, D. J.; Gong, X. G. Curvature effect on surface diffusion: The nanotube. The Journal of Chemical Physics 2001, $\overline{114}$, 10922-10926.

(50) Liu, L.; Chen, Z.; Wang, L.; Polyakova, E.; Taniguchi, T.; Watanabe, K.; Hone, J.; Flynn, G. W.; Brus, L. E. Slow gold adatom diffusion on graphene: Effect of silicon dioxide and hexagonal boron 
nitride substrates. Journal of Physical Chemistry B 2013, 117, 4305-4312.

(51) Neek-Amal, M.; Abedpour, N.; Rasuli, S.; Naji, A.; Ejtehadi, M. Diffusive motion of $\mathrm{C}_{60}$ on a graphene sheet. Physical Review E 2010, 82, 051605.

(52) Lohrasebi, A.; Neek-Amal, M.; Ejtehadi, M. Directed motion of $\mathrm{C}_{60}$ on a graphene sheet subjected to a temperature gradient. Physical Review E 2011, 83, 042601.

(53) Meiser, J.; Weindl, D.; Hiller, K. Complexity of dopamine metabolism. Cell Communication and Signaling 2013, 11, 34.

(54) Hamm, L. L.; Nakhoul, N.; HeringSmith, K. S. Acid-base homeostasis. Clinical Journal of the American Society of Nephrology 2015, 10, 2232-2242.

(55) National Center for Biotechnology Information Database: Dopamine. https://pubchem.ncbi.nlm.nih.gov/ compound/Dopamine.

(56) Nishihira, J.; Tachikawa, H. Theoretical Study on the Interaction Between Dopamine and its Receptor byab initioMolecular Orbital Calculation. Journal of theoretical biology 1997, 185, 157-163.

(57) Plimpton, S. Fast Parallel Algorithms for Short-Range Molecular Dynamics. Journal of Computational Physics 1995, 117, 1-19.

(58) Jorgensen, W. L.; Maxwell, D. S.; TiradoRives, J. Development and Testing of the OPLS All-Atom Force Field on Conformational Energetics and Properties of Organic Liquids. Journal of the American Chemical Society 1996, 118, 1122511236.

(59) Lazar, P.; Karlický, F.; Jurečka, P.; Kocman, M.; Otyepková, E.; Šafářová, K.; Otyepka, M. Adsorption of Small Organic Molecules on Graphene. Journal of the
American Chemical Society 2013, 135, 6372-6377.

(60) Björk, J.; Hanke, F.; Palma, C.-A.; Samori, P.; Cecchini, M.; Persson, M. Adsorption of Aromatic and Anti-Aromatic Systems on Graphene through $\pi-\pi$ Stacking. The Journal of Physical Chemistry Letters 2010, 1, 3407-3412.

(61) Bath, B. D.; Michael, D. J.; Trafton, B. J.; Joseph, J. D.; Runnels, P. L.; Wightman, R. M. Subsecond Adsorption and Desorption of Dopamine at Carbon-Fiber Microelectrodes. Analytical Chemistry 2000, 72, 5994-6002.

(62) Tsuzuki, S.; Honda, K.; Uchimaru, T.; Mikami, M.; Tanabe, K. Origin of attraction and directionality of the $\pi / \pi$ interaction: model chemistry calculations of benzene dimer interaction. Journal of the American Chemical Society 2002, 124, 104-112.

(63) Zhou, P.-P.; Zhang, R.-Q. Physisorption of benzene derivatives on graphene: critical roles of steric and stereoelectronic effects of the substituent. Physical Chemistry Chemical Physics 2015, 17, 12185-12193.

(64) Li, Z. Nanofluidics: An Introduction; CRC Press, 2018

(65) Weiss, L. B.; Dahirel, V.; Marry, V.; Jardat, M. Computation of the hydrodynamic radius of charged nanoparticles from nonequilibrium molecular dynamics. The Journal of Physical Chemistry B 2018, 122, 5940-5950.

(66) Haydukivska, K.; Blavatska, V.; Paturej, J. Universal size ratios of Gaussian polymers with complex architecture: radius of gyration vs hydrodynamic radius. Scientific Reports 2020, 10, 1-11.

(67) Lee, H.; Venable, R. M.; MacKerell Jr, A. D.; Pastor, R. W. Molecular dynamics studies of polyethylene oxide and polyethylene glycol: hydrodynamic 
radius and shape anisotropy. Biophysical journal 2008, 95, 1590-1599.

(68) Zwanzig, R.; Harrison, A. K. Modifications of the Stokes-Einstein formula. The Journal of chemical physics 1985, 83, 5861-5862.

(69) Kowert, B. A.; Turner II, R. M.; Caldwell, C. V. Diffusion of 1-alkenes and cyclohexene in alkane solvents. Chemical Physics 2008, 344, 114-120.

(70) Edward, J. T. Molecular volumes and the Stokes-Einstein equation. Journal of chemical education 1970, 47, 261.

(71) Evans, R.; Dal Poggetto, G.; Nilsson, M.; Morris, G. A. Improving the interpretation of small molecule diffusion coefficients. Analytical chemistry 2018, 90, 3987-3994.

(72) Chan, T.; Li, H.; Li, K. Effects of shapes of solute molecules on diffusion: A study of dependences on solute size, solvent, and temperature. The Journal of Physical Chemistry B 2015, 119, 15718-15728.

(73) Su, J. T.; Duncan, P. B.; Momaya, A.; Jutila, A.; Needham, D. The effect of hydrogen bonding on the diffusion of water in n-alkanes and n-alcohols measured with a novel single microdroplet method. The Journal of chemical physics 2010, $\underline{132}$, 044506.

(74) Heien, M. L.; Phillips, P. E.; Stuber, G. D.; Seipel, A. T.; Wightman, R. M. Overoxidation of carbon-fiber microelectrodes enhances dopamine adsorption and increases sensitivity. Analyst 2003, 128, 1413-1419.

(75) Cao, Q.; Lucktong, J.; Shao, Z.; Chang, Y.; Venton, B. J. Electrochemical treatment in $\mathrm{KOH}$ renews and activates carbon fiber microelectrode surfaces. Analytical and Bioanalytical Chemistry 2021, 1-10.
(76) Striolo, A. The mechanism of water diffusion in narrow carbon nanotubes. Nano Lett. 2006, 6, 633-639.

(77) Hirunsit, P.; Balbuena, P. B. Effects of confinement on water structure and dynamics: a molecular simulation study. The Journal of Physical Chemistry C 2007, 111, 1709-1715.

(78) Falk, K.; Sedlmeier, F.; Joly, L.; Netz, R. R.; Bocquet, L. Molecular Origin of Fast Water Transport in Carbon Nanotube Membranes: Superlubricity versus Curvature Dependent Friction. Nano Letters 2010, 10, 4067-4073.

(79) Barati Farimani, A.; Aluru, N. R. Spatial diffusion of water in carbon nanotubes: from fickian to ballistic motion. The Journal of Physical Chemistry B 2011, 115, 12145-12149.

(80) Zheng, Y.-g.; Ye, H.-f.; Zhang, Z.-q.; Zhang, H.-w. Water diffusion inside carbon nanotubes: mutual effects of surface and confinement. Phys. Chem. Chem. Phys. 2012, 14, 964-971.

(81) Shiratori, N.; Lee, K.; Miyawaki, J.; Hong, S.-H.; Mochida, I.; An, B.; Yokogawa, K.; Jang, J.; Yoon, S.-H. Pore structure analysis of activated carbon fiber by microdomain-based model. Langmuir 2009, 25, 7631-7637.

(82) Chen, B.; Perry, D.; Teahan, J.; McPherson, I. J.; Edmondson, J.; Kang, M.; Valavanis, D.; Frenguelli, B. G.; Unwin, P. R. Artificial Synapse: Spatiotemporal Heterogeneities in Dopamine Electrochemistry at a Carbon Fiber Ultramicroelectrode. ACS Measurement Science $\mathrm{Au} 2021$, 